\title{
Structure and mobility of cyclohexane as a solvent for Trans-Polyisoprene
}

\author{
Roland Faller ${ }^{a}$ \\ ${ }^{a}$ Department of Chemical Engineering, \\ University of Wisconsin, Madison, WI 53706-1691, USA \\ E-mail:faller@che.wisc.edu
}

November 1, 2018

Solutions of trans-polyisoprene in cyclohexane are investigated in atomistic detail at different compositions at two different temperatures. We investigate the influence of polymer concentration on the dynamics of the solvent molecules and the structure of the solvation shell. The double bonds along the polymer backbone are preferentially approached by the solvent molecules. The mobility of cyclohexane molecules decreases with increasing polymer concentration at ambient conditions. The reorientation of molecules becomes more anisotropic with concentration as the polymer hinders the reorientation of the molecular plane. At elevated temperatures the influence of the polymer is weaker and the reorientation of the solvent is more isotropic. Additionally, a fast and efficient way to set up atomistic simulations is shown in detail in which the initial simulations increase in length and in the simulation time-step. The excess energy from initial overlaps is removed by resetting the velocities at regular intervals.

\section{Introduction}

Polymeric solutions are important for the understanding of technologically important applications of polymers. Therefore they have been in the focus of molecular simulations over the recent decade. Many studies have been performed to investigate generic characteristics of polymer solutions by means of simple bead-spring models [1,2]. However, to investigate the behavior of a system on the local scale, fully atomistic simulations will be required. Several authors have dealt with atomistically detailed descriptions of polymer solutions [3 6 . But, there are only few studies how the polymer influences the solvent dynamics [7. 8]. Especially, the region of low weight fraction of polymer has not yet been studied. This contribution focuses on the liquid structure and dynamics of small solvent molecules in the neighborhood of oligomer chains in dilute solution. We use as an example transpolyisoprene dissolved in cyclohexane. Cyclohexane is a rather spherical organic molecule and experimentally known to be a good solvent for polyisoprene [9, 10]. Polyisoprene by itself is one of the most commonly used polymers. Its natural form, cautschuk, is highly abundant in nature and for technological purposes, synthetic variations are easily industrially polymerised from isoprene. Still, the solutions of such important materials are not yet completely understood from a microscopic viewpoint.

\section{Details of the System}

Systems containing one or two 15-mers $\left(\mathrm{C}_{75} \mathrm{H}_{122}\right)$ of trans-polyisoprene (PI) are simulated in a solution consisting of 250 or 500 molecules of cyclohexane $\left(\mathrm{C}_{6} \mathrm{H}_{12}\right)$. An overview over all the systems is given in Table 1, also the resulting densities in the simulations are shown in that figure. Details of the self-developed forcefields are found in ref. [11] for trans-polyisoprene and refs. [12, 13] for cyclohexane. The polyisoprene forcefield is in contrast to the original version enhanced with torsions for the methyl groups with a strength of $3.3 \mathrm{~kJ} / \mathrm{mol}$ per atom $(9.9 \mathrm{~kJ} / \mathrm{mol}$ per methyl group). The cyclohexane forcefield is augmented with torsion potentials for all the $\mathrm{C}-\mathrm{C}$ bonds. Each of them has a strength of $10 \mathrm{~kJ} / \mathrm{mol}$ in order to prevent random flipping between configurations. The cyclohexane molecule can occur in the so-called chair or boat configuration. They are distinguished if the connection between $\mathrm{C}_{1}$ and $\mathrm{C}_{4}$ intersects the plane defined by the other carbons (chair) or not (boat). All 
Table 1: Details of the different systems. $N_{P}$ is the number of oligomers $\left(\mathrm{C}_{75}\right)$ and $N_{C}$ the number of cyclohexane molecules. $c$ is the concentration in weight $\%$ polymer. $t_{\text {sim }}$ is the simulated time for the systems. Additionally the resulting densities $\rho$ are shown for the simulated temperatures.

\begin{tabular}{ccccccc}
\hline$\#$ & $N_{P}$ & $N_{C}$ & $\mathrm{c}$ & $t_{\text {sim }}$ & $\rho(300 \mathrm{~K})$ & $\rho(413 \mathrm{~K})$ \\
\hline 0 & 0 & 250 & $0.0 \%$ & $1 \mathrm{~ns}$ & $756.3 \mathrm{~kg} / \mathrm{m}^{3}$ & $627.4 \mathrm{~kg} / \mathrm{m}^{3}$ \\
1 & 1 & 250 & $4.6 \%$ & $2 \mathrm{~ns}$ & $764.2 \mathrm{~kg} / \mathrm{m}^{3}$ & $637.2 \mathrm{~kg} / \mathrm{m}^{3}$ \\
2 & 1 & 500 & $2.4 \%$ & $2 \mathrm{~ns}$ & $757.5 \mathrm{~kg} / \mathrm{m}^{3}$ & $629.2 \mathrm{~kg} / \mathrm{m}^{3}$ \\
3 & 2 & 500 & $4.6 \%$ & $2 \mathrm{~ns}$ & $762.5 \mathrm{~kg} / \mathrm{m}^{3}$ & $635.2 \mathrm{~kg} / \mathrm{m}^{3}$ \\
4 & 2 & 250 & $8.9 \%$ & $2 \mathrm{~ns}$ & $768.2 \mathrm{~kg} / \mathrm{m}^{3}$ & $643.5 \mathrm{~kg} / \mathrm{m}^{3}$ \\
\hline
\end{tabular}

simulations and analyses are performed using the YASP molecular dynamics package 14]. No charges are used throughout the simulations.

Both forcefields have been proven useful in recent simulations. The polyisoprene forcefield was used to investigate the dynamics of oligomer melts [1]] whereas the cyclohexane model was used in simulations of pure cyclohexane and its mixtures with cyclohexene at ambient conditions [13].

\section{How to set up and equilibrate an atomistic simulation}

In atomistic simulations, the way a system is initially set up and equilibrated is crucial. Thus, we describe the equilibration procedure for our systems in detail. For system 1 (one polymer chain solvated by 250 cyclohexanes) the 250 cyclohexane molecules were put onto a regular fcc lattice in a cubic box of $4 \mathrm{~nm}$ side-length corresponding to a density of $\rho=545.8 \mathrm{~kg} / \mathrm{m}^{3}$. An $N V E$ simulation with a very short timestep of $10^{-3}$ fs was started. The timestep was increased from $10^{-3}$ fs to 1 fs in three steps; in each step it was multiplied by a factor of 10 . Each simulation was run for 1000 steps at constant volume and energy. The velocities of the particles were reset from a Maxwell-Boltzmann distribution corresponding to $300 \mathrm{~K}$ before each run. The polyisoprene molecule was inserted at random, and the pre-equilibration procedure restarted. This time the initial timestep was set to $10^{-6}$ fs and the steps were increased by factors of 100 , only the last two increases were by factors of 10 . The initial polyisoprene conformation was taken from the $\mathrm{C}_{75}$ chains of the melt system 3 of ref. [11]. In the rare case that one of the short simulations failed because of a non-convergence of the SHAKE algorithm [15] an additional reset of the velocities was applied.

This method of pre-equilibration is very fast for a fully atomistic system. It avoids the use of unphysical softcore simulations. By resetting the velocities to the desired temperature periodically, the system moves very efficiently away from its initial high energy. One can randomly insert the solute if the density is low and the timestep sufficiently short. The very short simulations at short timesteps have the additional advantage that not too much energy is transferred to internal degrees of freedom which would lead to longer simulations in later equilibration stages.

After the simulation was running stable under $N V E$ conditions at a timestep of $1 \mathrm{fs}$, the Weak-Coupling themostat [16] was switched on. 10 ps later the isotropic Weak-Coupling constant pressure routine was switched on to compress the system to the correct density. The compressibility was set to the experimental value of $1.12 \mathrm{GPa}^{-1}$ of pure cyclohexane [17]. Coupling times for pressure and temperature were $\tau_{T}=0.2 \mathrm{ps}$ and $\tau_{p}=2.0 \mathrm{ps}$ respectively. The first constant pressure run, however, started with $\Delta t=0.1 \mathrm{fs}$ and $\tau_{p}=200 \mathrm{ps}$. A stepwise increase of the timestep and decrease of the coupling times was performed until the simulations under production conditions were possible $\left(\tau_{T}=0.2 \mathrm{ps}, \tau_{p}=2.0 \mathrm{ps}, \Delta t=2 \mathrm{fs}\right)$.

A second system was produced the same way but independently with another 15-mer from the melt simulations. These two systems were merged to form the systems with two polymers. The system with only 250 cyclohexane molecules and 2 polymers was produced by taking out every second cyclohexane and letting the system relax to the correct density in an $N p T$ simulation.

After proper relaxation at the correct timestep using temperature and pressure coupling, the production runs were started. The relaxation lasted longer than the correlation time for the end-to-end length of the polymer to lose the memory of the melt structure. The production runs lasted up to 5 ns. The reorientation correlation time of the chains was around 2 ns. Configurations were saved every 1000 steps (i.e. 2 ps). The simulations were performed at ambient conditions $(T=300 \mathrm{~K}, p=101.3 \mathrm{kPa})$ and at $T=413 \mathrm{~K}, p=101.3 \mathrm{kPa}$. The higher temperature was chosen for consistency with simulations of solutions of polyisoprene in toluene [3] although cyclohexane and toluene are gases at that temperature in nature. This vaporization transition is not reproduced by the force-field. The cutoff for the Lennard-Jones interaction was set to $0.9 \mathrm{~nm}$ and for the neighbor list [18] to $1.0 \mathrm{~nm}$. The neighbor-list was updated every 10 timesteps and the total momentum of the box was reset to 


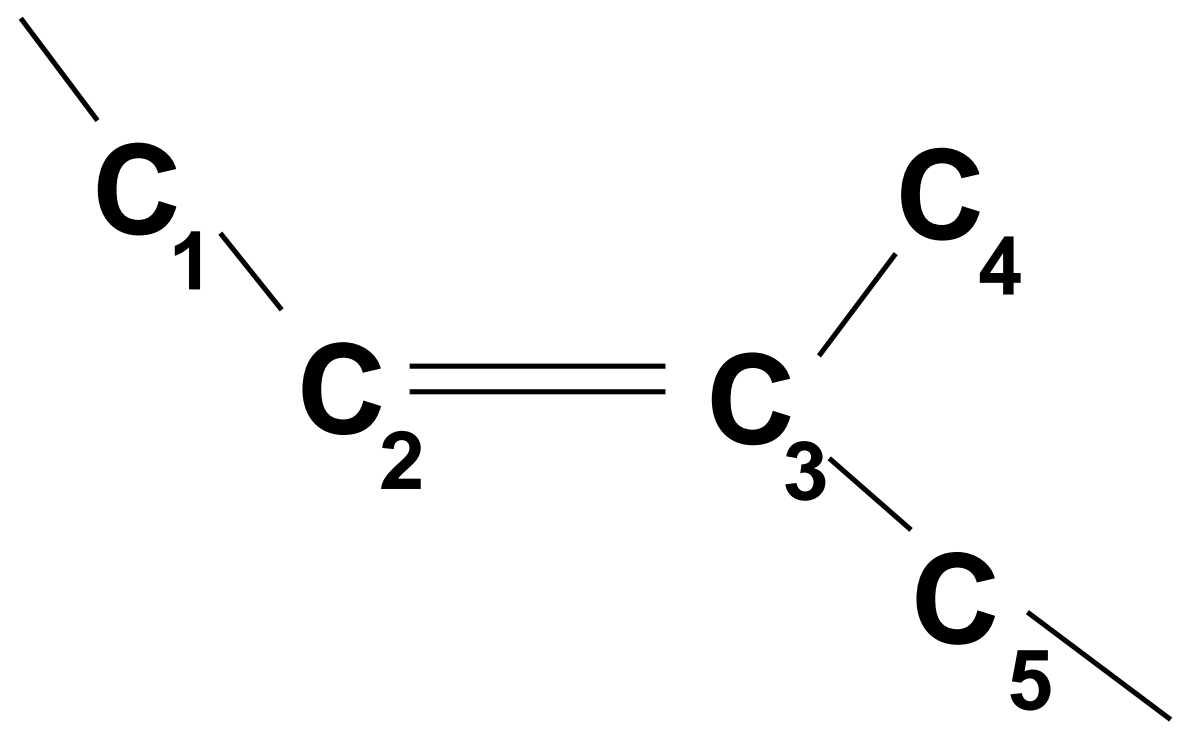

Figure 1: Sketch of the carbons of a trans-polyisoprene monomer indicating the numbers they are referred to in this work
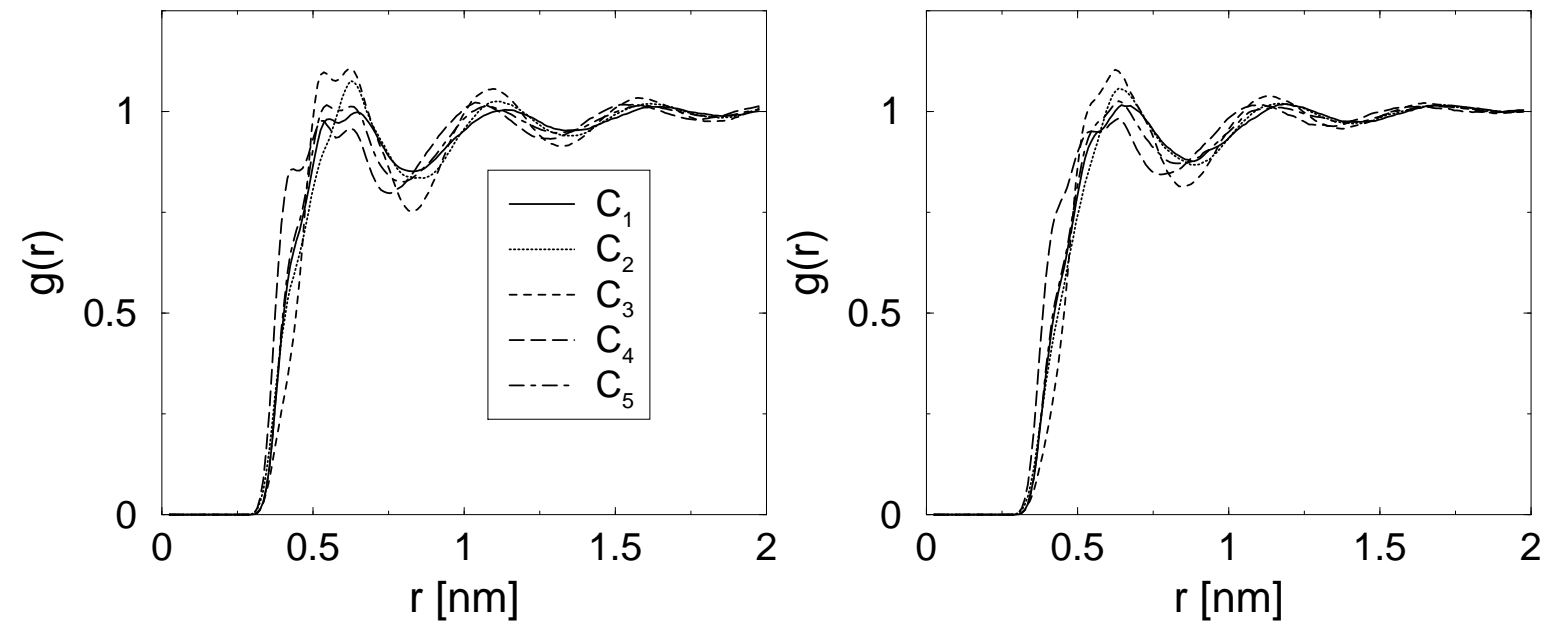

Figure 2: Radial distribution functions of the polymer carbons against the cyclohexane carbons for the system with 2 polymers and 500 cyclohexanes. a) $300 \mathrm{~K} \mathrm{b)} \mathrm{413K.} \mathrm{The} \mathrm{legend} \mathrm{of} \mathrm{figure} \mathrm{a)} \mathrm{applies} \mathrm{to} \mathrm{both} \mathrm{parts.}$

zero every 1000 timesteps. A pure cyclohexane system was simulated to investigate the influences of the newly added torsions in the forcefield in comparison to ref. 13 .

\section{Statics and Dynamics of Cyclohexane solvating Polyisoprene}

In this section the carbons along the polymer chain are referred to in the following way: $\mathrm{C}_{1}$ is the non-methyl end of the monomer. $\mathrm{C}_{2}$ and $\mathrm{C}_{3}$ are connected by the double bond. $\mathrm{C}_{2}$ is connected to $\mathrm{C}_{1}, \mathrm{C}_{4}$ is the methyl carbon, and $\mathrm{C}_{5}$ is between $\mathrm{C}_{3}$ and $\mathrm{C}_{1}$ (cf. Fig. 11).

The first question we want to address is the way the cyclohexane molecules solvate polyisoprene. Therefore we investigate the number of cyclohexane carbons close to the five distinct carbons along the chain. We do not distinguish between different concentrations here. To avoid end effects, the end monomers are excluded from the calculation. The resulting radial distribution functions are shown in Figure 2 .

The main difference between the two temperatures is that for $\mathrm{T}=300 \mathrm{~K}$ the neighbor peak is a double peak for some of the carbons. The two sub-peaks are about $0.07 \mathrm{~nm}$ apart. This structure does not persist at the elevated temperature. The overall height of the peaks is, however, unchanged. This indicates that the number of cyclohexanes solvating a monomer is not temperature dependent. The arrangement of the solvent molecules, however, is. $\mathrm{C}_{4}$ which is the side group, can come into closest contact with the solvent visible at the shoulder at 
Table 2: Motion of cyclohexane in the presence of polyisoprene. The error of the diffusion coefficients was estimated by the anisotropy of the mean-squared displacements. The errors of the reorientation times were estimated by the scatter of the times of the different vectors. The upper half of the table corresponds to $\mathrm{T}=300 \mathrm{~K}$, the lower half to $\mathrm{T}=413 \mathrm{~K}$. The $x-y$ notation means that the system contains $x$ polymer chains and $y$ solvent molecules. The differences between the $1-250$ and the $2-500$ system can be used as an estimate of the simulation error or finite size effects as both these systems are at the same concentration. These effects are on the order of less than $5 \%$.

\begin{tabular}{ccccc}
\hline conc. & $\mathrm{D}\left[10^{-5} \mathrm{~cm}^{2} / \mathrm{s}\right]$ & $\tau_{\|}[\mathrm{ps}]$ & $\tau_{\perp}[\mathrm{ps}]$ & $\tau_{\perp} / \tau_{\|}$ \\
\hline pure & $0.78 \pm 0.09$ & $3.2 \pm 0.4$ & $4.4 \pm 0.1$ & 1.38 \\
$1-500$ & $0.76 \pm 0.02$ & $3.84 \pm 0.06$ & $4.69 \pm 0.05$ & 1.22 \\
$1-250$ & $0.66 \pm 0.03$ & $3.65 \pm 0.08$ & $4.90 \pm 0.06$ & 1.34 \\
$2-500$ & $0.71 \pm 0.03$ & $3.83 \pm 0.12$ & $5.08 \pm 0.14$ & 1.32 \\
$2-250$ & $0.65 \pm 0.03$ & $3.84 \pm 0.04$ & $5.24 \pm 0.06$ & 1.36 \\
\hline \hline pure & $5.2 \pm 0.2$ & $1.4 \pm 0.1$ & $1.45 \pm 0.04$ & 1.03 \\
$1-500$ & $5.6 \pm 0.5$ & $1.30 \pm 0.02$ & $1.43 \pm 0.02$ & 1.1 \\
$1-250$ & $5.0 \pm 0.4$ & $1.38 \pm 0.02$ & $1.41 \pm 0.02$ & 1.02 \\
$2-500$ & $5.3 \pm 0.3$ & $1.32 \pm 0.02$ & $1.42 \pm 0.02$ & 1.08 \\
$2-250$ & $4.7 \pm 0.3$ & $1.35 \pm 0.04$ & $1.46 \pm 0.02$ & 1.08 \\
\hline
\end{tabular}

$r \approx 0.4 \mathrm{~nm}$. It was also seen in the melt investigations that this carbon is most exposed [11]. All carbons exhibit a peak at the first solvation shell at $r \approx 0.55-0.6 \mathrm{~nm}$. A second $(r \approx 1.1 \mathrm{~nm})$ and third $(r \approx 1.55 \mathrm{~nm})$ solvation shell are visible. The first peak is strongest for the two double bonded carbons. They are not very strongly shielded by hydrogens and are therefore rather easily accessible. This is in contrast to the melt simulations where the lacking mobility of these carbons prevented a close approach to neighboring chains [11]. As the mobility of the solvent is much higher than that of the polymer, the differences in mobility of the polymer atoms is not important. At the higher temperature, the second and third solvation shell are slightly shifted to larger distances. The third solvation shell is rather broad.

The cyclohexane diffusion is found to be completely isotropic and the molecules have diffused several times their gyration radius. The diffusion coefficient is measured by fitting the mean-squared displacement of the center-of-mass of the cyclohexane molecules to $\left\langle\vec{r}^{2}\right\rangle=6 D t$. The results are found in Table 2. The diffusion coefficient for pure cyclohexane in this model is found to be $0.78 \times 10^{-5} \mathrm{~cm}^{2} / \mathrm{s}$ at $T=300 \mathrm{~K}$. The value of $0.89 \times 10^{-5} \mathrm{~cm}^{2} / \mathrm{s}$ reported in reference [13] is about $15 \%$ higher. This is probably due to the missing internal torsions in that work which make it easier for the molecule to squeeze through small voids. The diffusivity of cyclohexane decreases with increasing polymer concentration. The polymer molecules can be viewed as obstacles for the solvent. This decrease is opposite to the pronounced increase of cyclohexane mobility in the presence of the smaller cyclohexene [13]. We can thus conclude that the mobility of cyclohexane depends strongly on the size and concentration of the molecules with which it is mixed.

Pure cyclohexane reorients faster in the plane defined by the carbon ring than the plane flips. To investigate the reorientation behavior in the presence of the polymer, we measure the two principal reorientation times of an oblate molecule, i.e. $\tau_{\|}$measures the reorientation of vectors connecting carbons across the ring, whereas $\tau_{\perp}$ is the characteristic time for rotating the axis normal to the plane. This axis is defined in the simulations as cross-product of two $\mathrm{C}-\mathrm{C}$ vectors across the ring. The former measures the reorientation of the molecule in its plane, whereas the latter measures the reorientation of the plane. In an earlier study, we found that pure cyclohexane, as well as cyclohexane in a mixture with cyclohexene, reorients faster in-plane than the plane reorients [13. This is expected for a dense liquid; in the gas-phase, one expects this order to be reversed due to the moments of inertia. The reorientation times presented in Table 2 were derived by an exponential fit to the first $25 \mathrm{ps}$ of the correlation function at $\mathrm{T}=300 \mathrm{~K}(10 \mathrm{ps}$ for $\mathrm{T}=413 \mathrm{~K})$. These fits reproduced the curves very accurately. The reorientation times are measured as correlation times of the first Legendre polynomial $P_{1}(t)=\langle\vec{u}(t) \vec{u}(0)\rangle$. The resolution and accuracy is better than for $P_{2}(t)$ as the corresponding times are longer. $\vec{u}(t)$ is the unit vector we are monitoring. We find signs for Debye rotation $T_{1}=3 T_{2}$, however, as $T_{2}$ is very short this cannot be completely decided. The cyclohexane model used in this work is slightly more anisotropic than its predecessor without internal torsions. The in-plane reorientation is exactly the same as in the old model [13]. The reorientation of the plane is about $10 \%$ slower as in the model lacking internal torsions.

The in-plane reorientation time is practically unaffected by the concentration of the polymer except for pure cyclohexane, whereas the reorientation of the plane becomes slower with increasing concentration. As the inplane reorientation does not need any other molecule to make room for the transition to occur, the opposite is 


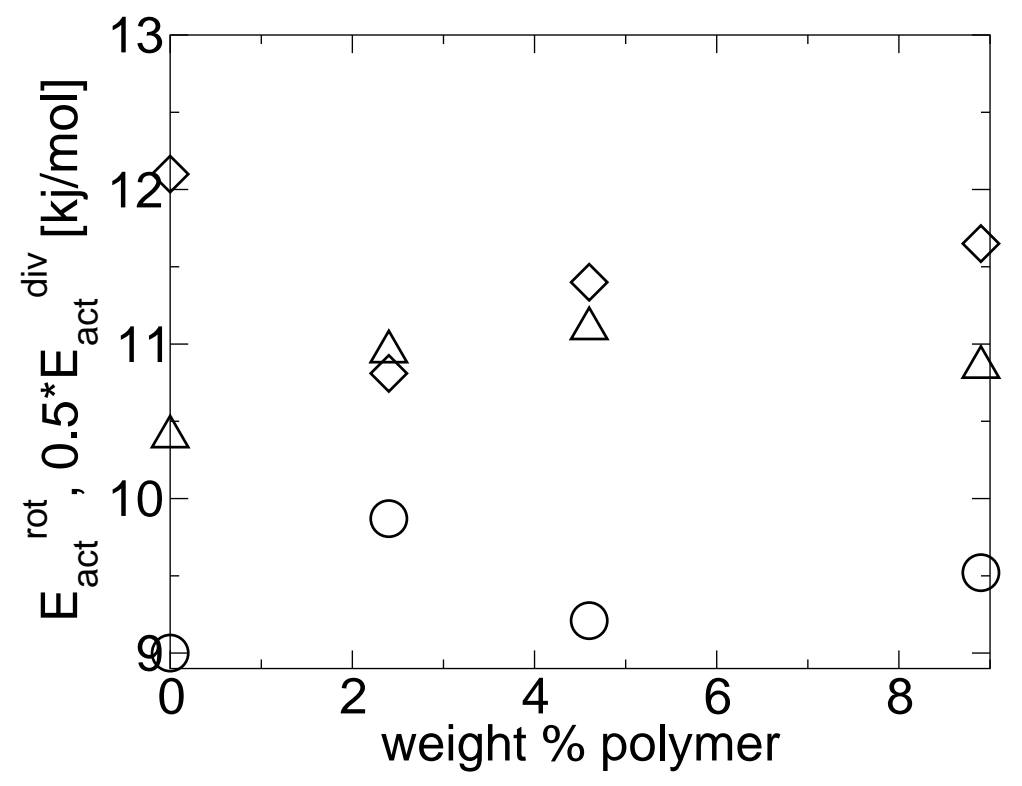

Figure 3: Estimated activation energies for the rotation in the plane (diamonds) and the plane flip (circles) depending on polymer concentration. Additionally the activation energy for the diffusion (triangles) is shown. The values for diffusion are divided by a factor of 2 .

the case for the plane-flip. Therefore the polymers act as obstacles to the molecules in their neighborhood and make their dynamics more anisotropic as concentration increases. Pure cyclohexane appears to be a different case as it is rather strongly anisotropic; this manifests itself in a clearly faster in-plane reorientation than all the other cases. This suggests that the in-plane reorientation of the molecules close to the polymer is hindered by the chain. This effect is similar in strength for all concentrations.

At the elevated temperature, the reorientation times are clearly shorter. The anisotropy at this temperature becomes weaker, and in the range of concentrations here, it does not depend systematically on polymer content. Assuming an Arrhenius behavior, we can estimate activation energies for the different rotations and the diffusion; they are shown in Figure 3. Note that the activation energies for the diffusion are divided by a factor of two. These activation energies are on the order of $E_{a c t}=10 \mathrm{~kJ} / \mathrm{mol}$ for the rotations and on the order of $20 \mathrm{KJ} / \mathrm{mol}$ for the diffusion and are only weakly dependent on concentration. We see that the in-plane reorientation is stronger temperature dependent than the plane flip. At very high temperatures an inversion of the reorientation order may therefore be expected in line with the moments of inertia. This explains the seemingly contradictory finding that the plane-flip is found to be slower in the simulated range but has the smaller activation energy. This is due to the stronger temperature dependence of the in-plane reorientation.

The increasing anisotropy of reorientation with concentration has been reported for two other systems at higher concentrations. It has been found for water as solvent of polyvinylacohol [8] and benzene as solvent of polystyrene [7].

The diffusion is much stronger temperature dependent than both the rotations. This can be understood by the fact that for a molecule to diffuse the neighborhood has to give way in a much stronger fashion, i.e. the whole neighborhood has to rearrange whereas for rotation the particle can in principle stay in place.

\section{Conclusion}

Altogether, we showed that the increasing polymer concentration has a decelerating effect on the dynamics of the cyclohexane solvent molecules. The polymer chains are obstacles to the cyclohexane motion and make their reorientation more anisotropic. Additionally we see, in comparison with the model lacking internal torsions, that even for molecules as small and rigid as cyclohexane internal torsion potentials have an effect on the overall dynamics.

The simulations presented here were additionally used as starting points for coarse-graining simulations with 
investigations of the chain statics and dynamics [19].

\section{Acknowledgments}

The author thanks Kevin van Workum for a critical reading of the manuscript. Financial support by the Emmy-Noether Program of the German Research Foundation (DFG) is gratefully acknowledged.

\section{References}

[1] B. Dünweg and K. Kremer, Phys. Rev. Letters, 1991, 66(23), 2996-2999.

[2] P. Ahlrichs and B. Dünweg, J. Chem. Phys., 1999, 111(17), 8225-8239.

[3] N. E. Moe and M. D. Ediger, Macromolecules, 1995, 28, 2329-2338.

[4] A. Perico, N. E. Moe, and M. D. Ediger, J. Chem. Phys., 1998, 108(3), 1245-1252.

[5] R. Witt, L. Sturz, A. Dölle, and F. Müller-Plathe, J. Phys. Chem. A, 2000, 104(24), 5716-5725.

[6] O. Borodin, D. Bedov, and G. D. Smith, Macromolecules, 2001, 34(16), 5687-5693.

[7] F. Müller-Plathe, Chem Phys Lett, 1996, 252(5-6), 419-424.

[8] F. Müller-Plathe, J Chem Phys, 1998, 108(19), 8252-8263.

[9] Y. Tsunashima, M. Hirata, N. Nemoto, and M. Kurata, Macromolecules, 1988, 21(4), 1107-1117.

[10] Y. Einaga, Prog. Polym. Sci., 1994, 19(1), 1-28.

[11] R. Faller, F. Müller-Plathe, M. Doxastakis, and D. Theodorou, Macromolecules, 2001, 34(5), 1436-1448.

[12] R. Faller, H. Schmitz, O. Biermann, and F. Müller-Plathe, J. Comput. Chem., 1999, 20(10), 1009-1017.

[13] H. Schmitz, R. Faller, and F. Müller-Plathe, J. Phys. Chem. B, 1999, 103(44), 9731-9737.

[14] F. Müller-Plathe, Comput. Phys. Commun., 1993, 78(1-2), 77-94.

[15] J.-P. Ryckaert, G. Cicotti, and H. J. C. Berendsen, J. Comput. Phys., 1977, 23(3), 327-341.

[16] H. J. C. Berendsen, J. P. M. Postma, W. F. van Gunsteren, A. DiNola, and J. R. Haak, J. Chem. Phys., 1984, 81(8), 3684-3690.

[17] ed. D. R. Lide, CRC handbook of chemistry and physics, CRC Press, Boca Raton, 76 ed., 1995.

[18] M. P. Allen and D. J. Tildesley, Computer Simulation of Liquids, Clarendon Press, Oxford, 1987.

[19] R. Faller and D. Reith, submitted to Macromolecules, 2002. 